\title{
Optimal Signaling Mechanisms in Unobservable Queues with Strategic Customers
}

\author{
DAVID LINGENBRINK, Cornell University \\ KRISHNAMURTHY IYER, Cornell University
}

We study the problem of optimal information sharing in the context of a service system. In particular, we consider an unobservable single server queue offering a service at a fixed price to a Poisson arrival of delaysensitive customers. The service provider can observe the queue, and may share information about the state of the queue with each arriving customer. The customers are Bayesian and strategic, and incorporate any information provided by the service provider into their prior beliefs about the queue length before making the decision whether to join the queue or leave without obtaining service. We pose the following question: which signaling mechanism and what price should the service provider select to maximize her revenue?

We formulate this problem as an instance of Bayesian persuasion in dynamic settings. The underlying dynamics make the problem more difficult because, in contrast to static settings, the signaling mechanism adopted by the service provider affects the customers' prior beliefs about the queue (given by the steady state distribution of the queue length in equilibrium). The core contribution of this work is in characterizing the structure of the optimal signaling mechanism. We summarize our main results as follows.

(1) Structural characterization: Using a revelation-principle style argument, we find that it suffices to consider signaling mechanisms where the service provider sends a binary signal of "join" or "leave", and under which the equilibrium strategy of a customer is to follow the service provider's recommended action.

(2) Optimality of threshold policies: For a given fixed price for service, we use the structural characterization to show that the optimal signaling mechanism can be obtained as a solution to a linear program with a countable number of variables and constraints. Under some mild technical conditions on the waiting costs, we establish that there exists an optimal signaling mechanism with a threshold structure, where service provider sends the "join" signal if the queue length is below a threshold, and "leave" otherwise. (In addition, at the threshold, the service provider randomizes.) For the special case of linear waiting costs, we derive an analytical expression for the optimal threshold in terms of the two branches of the Lambert-W function.

(3) Revenue comparison: Finally, we show that with the optimal choice of the fixed price and using the corresponding optimal signaling mechanism, the service provider can achieve the same revenue as with the optimal state-dependent pricing mechanism in a fully-observable queue. This implies that in settings where state-dependent pricing is not feasible, the service provider can effectively use optimal signaling (with the optimal fixed price) to achieve the same revenue.

CCS Concepts: •Mathematics of computing $\rightarrow$ Queueing theory; Markov processes; Linear programming; •Theory of computation $\rightarrow$ Algorithmic mechanism design;

Additional Key Words and Phrases: Bayesian persuasion; optimal pricing in queues; throughput optimization; threshold strategies

The second author gratefully acknowledges support from the NSF under grants CMMI-1462592 and CMMI-1633920. The full version of this paper is available at https://ssrn.com/abstract=2964093.

Contact author's address: dal299@cornell.edu.

Permission to make digital or hard copies of part or all of this work for personal or classroom use is granted without fee provided that copies are not made or distributed for profit or commercial advantage and that copies bear this notice and the full citation on the first page. Copyrights for third-party components of this work must be honored. For all other uses, contact the owner/author(s).

(c) 2017 Copyright held by the owner/author(s).

DOI: http://dx.doi.org/10.1145/3033274.3085135

EC'17, June 26-30, 2017, Cambridge, MA, USA. ACM ISBN 978-1-4503-4527-9/17/06. 